\title{
A mouse model of asthma-chronic obstructive pulmonary disease overlap induced by intratracheal papain
}

\author{
Kensuke Fukuda ${ }^{1}$, HIROTAKA MATSUZAKI ${ }^{1}$, Yu Mikami ${ }^{1}$, Kosuke Makita ${ }^{1}$, Kazuko \\ Miyakawa $^{1}$, Naoya Miyashita ${ }^{1}$, Keisuke Hosoki ${ }^{1}$, Takashi Ishii ${ }^{1}$, Satoshi Noguchi ${ }^{1}$, Hirokazu \\ Urushiyama $^{1}$, Masafumi Horie ${ }^{2}$, Akihisa Mitani ${ }^{1}$, Yasuhiro Yamauchi ${ }^{3}$, Eri Shimura ${ }^{4}$, \\ Susumu Nakae ${ }^{5}$, Akira Saito ${ }^{1}$, Takahide Nagase ${ }^{1}$, and Yoshihisa Hiraishi ${ }^{1}$ \\ ${ }^{1}$ The University of Tokyo \\ ${ }^{2}$ Osaka University \\ ${ }^{3}$ Tokyo University of Foreign Studies \\ ${ }^{4}$ Juntendo University - Sakura Campus \\ ${ }^{5}$ The Institute of Medical Science, The University of Tokyo
}

May 20, 2020

\section{Title}

A mouse model of asthma-chronic obstructive pulmonary disease overlap induced by intratracheal papain

To the Editor:

Asthma-chronic obstructive pulmonary disease (COPD) overlap (ACO) is recognised as a distinct clinical disorder that can be differentiated from asthma or COPD alone, owing to the rapid disease progression, frequent exacerbations, and increased comorbidities. ${ }^{1} \mathrm{~A}$ major impediment to the elucidation of mechanisms underlying ACO pathogenesis has been the lack of development and detailed validation of appropriate animal models. Administration of papain, a cysteine protease from the fruit of the plant species Carica papaya, was shown to induce acute/subacute allergic airway inflammation (AAI) via type 2 responses. $^{2}$ Results from previous mouse model experiments also indicated that short-term exposure to papain induces emphysema. ${ }^{3}$ We hypothesised that weekly intratracheal aerosol administration of papain could induce a clinically-feasible murine ACO model that features concurrent emphysema, persistent AAI, and airway hyperresponsiveness. Further, we verified its validity with multifaceted analysis including comprehensive cytokine analyses, neutrophil gelatinase-associated lipocalin (NGAL) measurement, and long-term analyses.

We first validated the model with respect to pulmonary emphysema. Porcine pancreatic elastase (PPE) has been used to induce murine models of COPD. ${ }^{4}$ We intratracheally administered PPE or papain using a microsprayer (Figure 1A). The changes in body weight detected among the mice in the PPE- or papaintreated groups were not significantly different from the controls (Figure S1). Both PPE- and papain-treated groups showed increased inspiratory capacity/weight and dynamic compliance (Figure 1B); representative lung histology revealed PPE- and papain-induced emphysema (Figure 1C). The mean linear intercept was significantly increased in the PPE- and papain-treated groups (Figure 1D). As such, we concluded that both PPE and papain induced emphysema. Furthermore, goblet cell hyperplasia was detected exclusively among mice in the papain-treated group (Figure 1E). Consistent with this finding, whole lung RNA preparations from the papain- (but not PPE-) treated mice exhibited increased expression of transcript encoding Muc5ac (Figure S2).

We then evaluated asthmatic features associated with papain administration. Representatives of bron- 
choalveolar lavage fluid (BALF) cytospin preparations are shown in Figure 1F. The total number of cells, macrophages, and eosinophils were significantly increased in the papain-treated group (Figure 1G). The papain-treated group exhibited higher airway responsiveness to increasing doses of methacholine compared to other groups (Figure 1H); no differences in baseline airway resistance were observed (Figure 1B). Whole lung homogenates of the papain-treated mice revealed increased expression levels of Eotaxin1 and Eotaxin2 (Figure S2). Thus, our papain-treated model had both COPD-associated and asthmatic features and was considered to be an appropriate model for ACO.

For more detailed analyses, multiple cytokines/chemokines in the BALF were quantitatively detected (Figure 2A; cytokine array data are shown in Table S1). Regarding macrophage/neutrophil-related cytokines/chemokines, macrophage colony-stimulating factor (M-CSF), keratinocyte-derived chemokine (KC), and IL-6 were detected in BALF from the papain-treated mice only. With regard to type 2 inflammationassociated cytokines, IL-4 levels were not significantly different among the groups while IL-5 and IL-13 levels were detectable only in the papain-treated mice. Collectively, our ACO model reproduced the characteristics of both asthmatic and COPD-associated airway inflammation. IL-33 concentrations were not significantly different among the groups (Figure S3). The levels of total IgE, a serum marker used to define asthma endotypes ${ }^{5}$ were significantly higher in the papain-treated mice (Figure 2B). Significantly higher levels of NGAL were detected in the BALF of papain-treated mice; NGAL has been identified as a promising biomarker for clinical evaluation of $\mathrm{ACO}^{6}$ (Figure 2C).

We also provide a more long-term ( 8 weeks; day 56) assessment of the papain-treated mice (Figure 2D). BALF eosinophils remained significantly elevated, a finding that indicates prolonged eosinophilic inflammation in response to papain (Figure 2E). Inspiratory capacity/weight and lung compliance also remained elevated at the 8-week time point (Figure 2F), as did emphysema-associated lung pathology (Figure 2G). ELISA was notable for elevated serum levels of total and papain-specific IgE at both 4 and 8 weeks (Figure $2 \mathrm{H}$ ). Taken together, our papain-induced model of ACO included prolonged eosinophilic type 2 inflammation along with emphysema. The papain-treated group at both 4 and 8 weeks similarly exhibited higher levels of NGAL in the BALF (Figure 2I).

Polyinosinic:polycytidylic acid [poly(I:C)] is a form of dsRNA that acts as a TLR3 agonist and is used to mimic viral infection-induced exacerbations of airway inflammation. ${ }^{7}$ In our poly(I:C)-induced exacerbation models (Figure S4A), neutrophil/macrophage-dominated inflammation was triggered while AAI was sustained in the ACO model (Figure S4B-E). We also found administration of poly(I:C) resulted in increased levels of NGAL both in serum and BALF (Figure S4F); of note, serum NGAL levels were increased only slightly above baseline by papain administration alone (Figure 2I). Since NGAL is expressed not only in the neutrophils but also in the respiratory epithelial cells, ${ }^{8}$ we hypothesised that poly(I:C)-induced elevations in NGAL were largely related to the influx of neutrophils; by contrast, NGAL resulting from papain administration alone may be result from inflammation-activated and/or damaged epithelial cells.

Paediatric asthma is a major predictor for the development of clinical ACO after middle age. ${ }^{9}$ As our model features administration of papain to mice at 5-6 weeks of age, our model would correspond to patients diagnosed with ACO associated with early-onset asthma. Specifically, our ACO model reflects early-onset, eosinophilic, high IgE, and Th2-dominant asthma phenotypes/endotypes. We recognise that emphysema in this model develops rapidly and at an earlier time point than what might be anticipated clinically and is not associated with smoking; these are both potential limitations of this model. However, this model is simpler and easier to use than most smoking models as it does not require several months of daily procedures and special equipment. ${ }^{4}$

To the best of our knowledge, this is the first established murine model of ACO that includes both prolonged emphysema and asthmatic features. Additionally, this is the first model of ACO that evaluated elevated levels of NGAL and as such parallels human clinical findings. The strong validation of this model includes results of comprehensive cytokine and NGAL measurements that reflect findings from clinical reports focused on ACO. ${ }^{6}$ Our murine model will be useful for elucidating the pathological processes associated with ACO and for identifying new diagnostic markers and therapeutic targets. 


\section{References}

1. Barnes PJ. Inflammatory endotypes in COPD. Allergy.2019;74:1249-1256.

2. Jacquet A, Robinson C. Proteolytic, lipidergic and polysaccharide molecular recognition shape innate responses to house dust mite allergens. Allergy. 2020;75:33-53.

3. Machado MN, Mazzoli-Rocha F, Casquilho NV, et al. Bone Marrow-Derived Mononuclear Cell Therapy in Papain-Induced Experimental Pulmonary Emphysema. Front Physiol. 2018;9:121.

4. Wright JL, Cosio M, Churg A. Animal models of chronic obstructive pulmonary disease. Am J Physiol Lung Cell Mol Physiol.2008;295:L1-L15.

5. McDowell PJ, Heaney LG. Different endotypes and phenotypes drive the heterogeneity in severe asthma. Allergy. 2020;75:302-310.

6. Iwamoto H, Gao J, Koskela J, et al. Differences in plasma and sputum biomarkers between COPD and COPD-asthma overlap. Eur Respir J.2014;43:421-429.

7. Akbarshahi H, Menzel M, Ramu S, Mahmutovic Persson I, Bjermer L, Uller L. House dust mite impairs antiviral response in asthma exacerbation models through its effects on TLR3. Allergy.2018;73:1053-1063.

8. Cowland JB, Borregaard N. Molecular characterization and pattern of tissue expression of the gene for neutrophil gelatinase-associated lipocalin from humans. Genomics. 1997;45:17-23.

9. Melen E, Guerra S, Hallberg J, Jarvis D, Stanojevic S. Linking COPD epidemiology with pediatric asthma care: Implications for the patient and the physician. Pediatr Allergy Immunol. 2019;30:589-597.

\section{Author names}

Kensuke Fukuda, ${ }^{1}$ Hirotaka Matsuzaki, ${ }^{* 1,2}$ Yu Mikami, ${ }^{1,3}$ Kosuke Makita, ${ }^{1,4}$ Kazuko Miyakawa, ${ }^{1}$ Naoya Miyashita, ${ }^{1}$ Keisuke Hosoki, ${ }^{1}$ Takashi Ishii, ${ }^{1,5}$ Satoshi Noguchi, ${ }^{1}$ Hirokazu Urushiyama, ${ }^{1}$ Masafumi Horie, ${ }^{6}$ Akihisa Mitani, ${ }^{1,5}$ Yasuhiro Yamauchi, ${ }^{7}$ Eri Shimura, ${ }^{8}$ Susumu Nakae, ${ }^{9}$ Akira Saito, ${ }^{1,5}$ Takahide Nagase, ${ }^{1}$ Yoshihisa Hiraishi ${ }^{1}$

\section{* corresponding author}

Hirotaka Matsuzaki, M.D., Ph.D.

Department of Respiratory Medicine, Graduate School of Medicine, The University of Tokyo, 7-3-1 Hongo, Bunkyo-ku, Tokyo 113-0033, Japan.

Phone: +81-3-3815-5411

E-mail: hmatsuzaki68@gmail.com

\section{Institution}

${ }^{1}$ Department of Respiratory Medicine, Graduate School of Medicine, the University of Tokyo, Tokyo, Japan.

${ }^{2}$ Centre for Epidemiology and Preventive Medicine, the University of Tokyo Hospital, Tokyo, Japan.

${ }^{3}$ Marsico Lung Institute/UNC Cystic Fibrosis Center, University of North Carolina at Chapel Hill School of Medicine, North Carolina, USA.

${ }^{4}$ Meakins-Christie Laboratories, Research Institute of the McGill University Health Centre, Quebec, Canada.

${ }^{5}$ Division for Health Service Promotion, the University of Tokyo, Tokyo, Japan.

${ }^{6}$ Department of Cancer Genome Informatics, Graduate School of Medicine, Osaka University, Osaka, Japan.

${ }^{7}$ Health Care Centre, Tokyo University of Foreign Studies, Tokyo, Japan.

${ }^{8}$ Department of Chemistry, Juntendo University School of Medicine, Chiba, Japan. 
${ }^{9}$ The Institute of Medical Science, The University of Tokyo, Tokyo, Japan.

\section{Acknowledgments}

We thank our laboratory members for the valuable discussions. This work was supported by the GSK

Japan Research Grant 2019 and the KAKENHI Grant Number 18K15946, Japan Society for the

Promotion of Science, the Ministry of Education, Culture, Sports, Science and Technology of Japan.

\section{Conflict of Interest}

The authors have declared that no conflict of interest exists.

Figure legends

Figure 1. Both porcine pancreatic elastase (PPE) and papain induced pulmonary emphysema, and papain reproduced the asthmatic features. (A) Protocol (B) Respiratory mechanics (C) Lung tissue stained with haematoxylin and eosin (HE; 20x) or (E) Alcian blue and periodic acid-Schiff (ABPAS; 20x) (D) Calculation of the mean linear intercept (F) Representative bronchoalveolar lavage fluid (BALF) cytospin preparations (50x); M, macrophage; E, eosinophil. (G) Differential cell counts of cytospin preparations $(\mathrm{H})$ Airway responsiveness to increasing doses of methacholine measured by subtracting the baseline from each airway resistance; ${ }^{*} p<0.05,{ }^{* *} p<0.01,{ }^{* * *} p<0.001 ;$ n.s. not significant.

Figure 2. Papain induced an increase in the macrophage/neutrophil- and type 2 inflammationassociated cytokines; allergic airway inflammation and emphysema persisted for 5 weeks after the final papain administration. (A) Cytokines in the BALF (B) Total serum IgE (C) Neutrophil gelatinase-associated lipocalin (NGAL) in the serum and BALF (D) Protocol (E) Differential cell counts from BALF cytospins (F) Respiratory mechanics (G) Lung tissue stained with HE; 20x. (H, I) Total and papain-specific IgE and NGAL; ${ }^{*} p<0.05,{ }^{* *} p<0.01,{ }^{* * *} p<0.001$; ND, not detected; n.s. not significant.

Figure S1. Body weights of mice in the PPE- and papain-treated groups were not significantly different from those treated with PBS control. Weights of mice treated with PPE $(\mathrm{n}=19)$, papain $(\mathrm{n}=27)$ or PBS $(\mathrm{n}=20)$ measured on days $0,7,14$, and 21 as in Fig. $1 \mathrm{~A} ;{ }^{* *} p<0.01$; n.s. not significant with two-way repeated measures ANOVA with Holm-Bonferroni correction.

Figure S2. Papain-induced mucus hypersecretion and allergic airway inflammation associated with elevated levels of transcript for Muc5ac, Muc5b, Eotaxin1 and Eotaxin2. Quantitative RT-PCR determination of whole lung mRNA encoding Muc5ac, Muc5b, Eotaxin1 and Eotaxin $2 ;{ }^{*} p<0.05$, ${ }^{* *} p<0.01,{ }^{* * *} p<0.001 ;$ n.s. not significant.

Figure S3. IL-33 in serum and BALF on day 25. Concentrations of IL-33 in serum and BALF measured by ELISA; n.s. not significant.

Figure S4. Administration of poly(I:C) augmented airway inflammation and NGAL in the PPE- and papain-treated mice; allergic airway inflammation was sustained in the papaintreated group. (A) Protocol (B) Representative BALF cytospins; L, lymphocyte; N, neutrophil; E, eosinophil (50x) (C) Differential cell counts from BALF cytospins (D) Lung tissue stained with HE (20x) (E) Cytokines in BALF (F) NGAL in the serum and BALF; ${ }^{*} p<0.05,{ }^{* *} p<0.01,{ }^{* * *} p<0.001$; n.s. not significant; ND, not detected.

Table S1. Cytokine array data. GM-CSF, Granulocyte macrophage colony-stimulating factor; IFN$\gamma$, Interferon gamma; IL, Interleukin; KC, Keratinocyte-derived chemokine (CXCL1); MCP-1, Monocyte chemotactic protein-1 (CCL2); M-CSF, Macrophage colony-stimulating factor; RANTES, Regulated on activation, normal $\mathrm{T}$ cell expressed and secreted (CCL5); TNF- $\alpha$, Tumour necrosis factor alpha; VEGF, Vascular endothelial growth factor. The data are presented as the mean $( \pm \mathrm{SEM}) . \mathrm{n}=3$ per group; ND, not detected. 

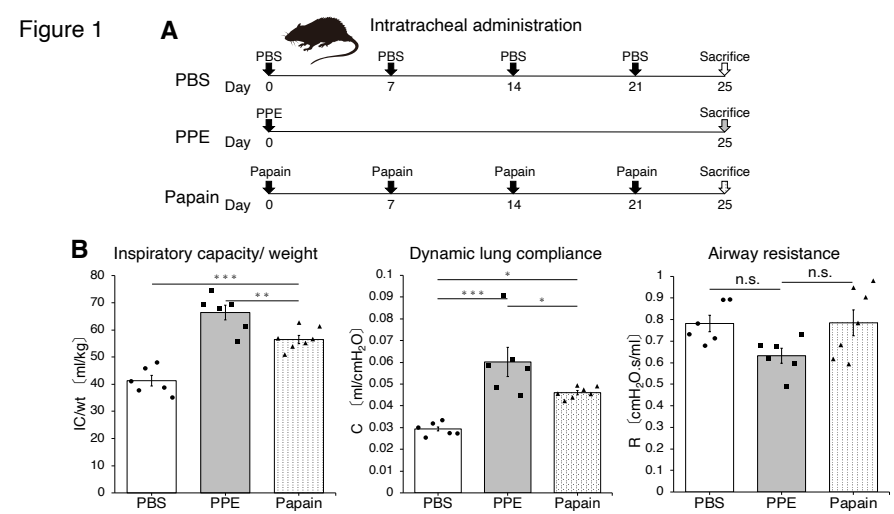

C
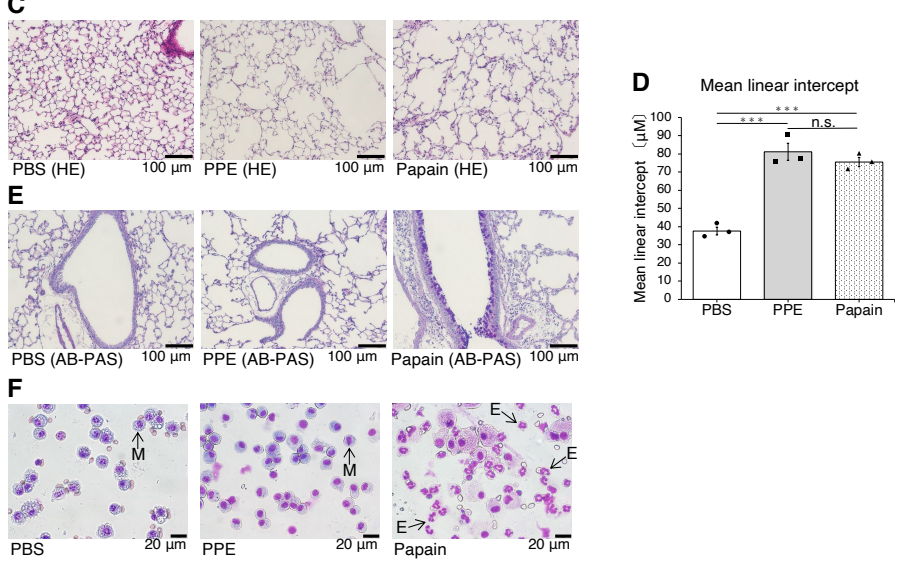

G

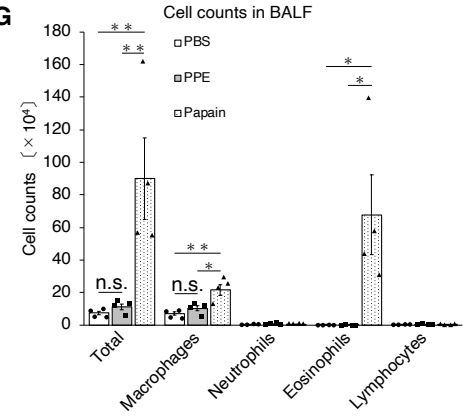

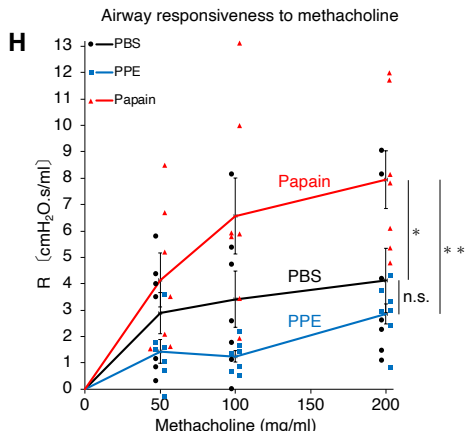


Figure 2
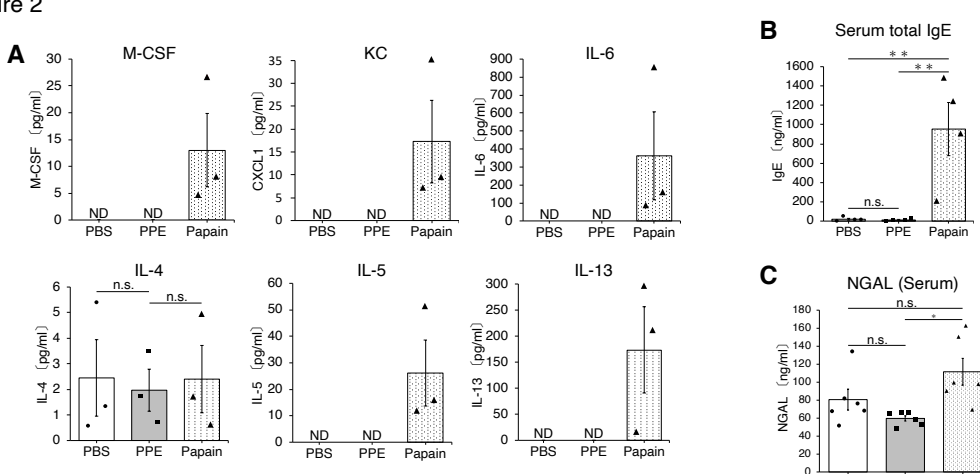

C NGAL (Serum)

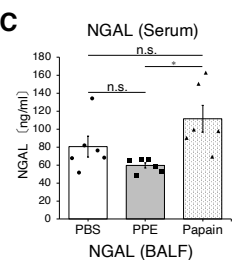

D
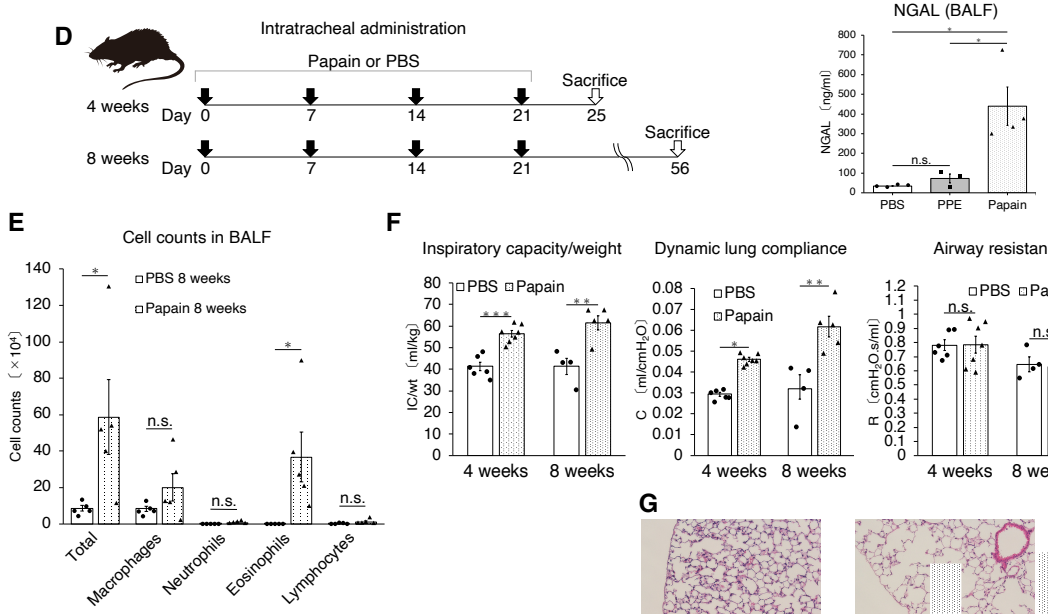

F

Inspiratory capacity/weight

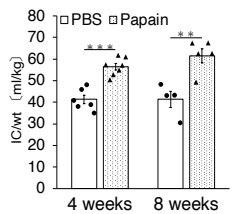

Dynamic lung compliance

Airway resistance

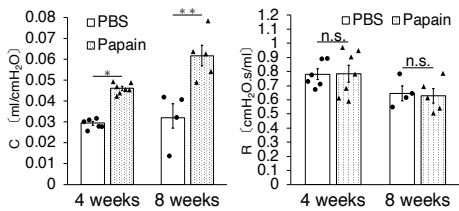

H

Serum total IgE Papain-specific IgE (Serum)

G
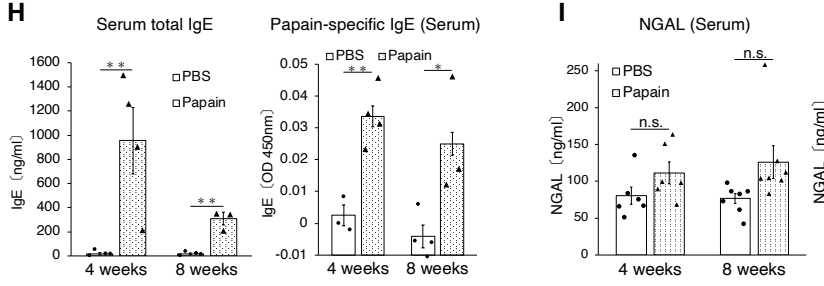

NGAL (BALF)

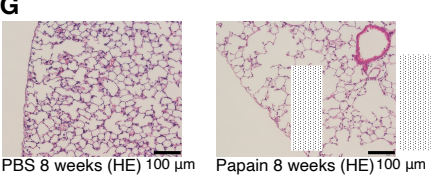

PBS 8 weeks (HE) $100 \mu \mathrm{m}$ Papain 8 weeks $(\mathrm{HE}) \overline{100} \mu \mathrm{m}$

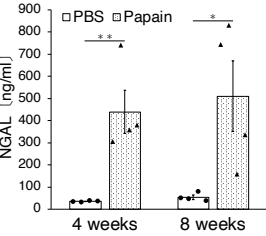

